\title{
THE SELECTION OF ALTERNATIVES TRAINING SHIP TO SUBSTITUTE KRI DEWARUCI FOR NAVAL ACADEMY CADETS USING THE ANALYTIC NETWORK PROCESS (ANP) METHOD
}

\author{
Sukmo Hadi Nugroho ${ }^{1}$, Adi Bandono ${ }^{2}$, Okol Sri Suharyo ${ }^{3}$, Raditya Novianto ${ }^{4}$ \\ ${ }^{1}$ Esa Unggul University, Jakarta Indonesia \\ ${ }^{2,3,4}$ Indonesian Naval Technology College, STTAL, Surabaya Indonesia
}

\begin{abstract}
Republic of Indonesia Ship (KRI) Dewaruci is a Naval Academy Cadets' (AAL) training ship that is old. This condition is very susceptible to various threats of accidents while carrying out voyages around the world to cruise the AAL Kartika Jala Krida Kadet. The government has planned to replace KRI Dewaruci with a new training ship. This study aims to determine the selection of new prospective makers training ship by the Analytic Network Process (ANP) method. This ANP method is used because the existing data have a relationship among the criteria and the relationship between criteria and sub-criteria. In alternative selection, there are two main criteria, namely operational requirements criteria with four sub-criteria: security, geographical conditions, skills training, transfer of technology and technical requirement criteria with five sub-criteria: machinery, navigation, training equipment, platform, masts, and sails. The results of this study are the alternative priorities for new training ship replacing the best KRI Dewaruci and also the priority of the main/critical sub-criteria. The biggest alternative score value is a training ship made by Piere Shipyard made in Spain with a score of 0.50259.
\end{abstract}

Keywords: Analytic Network Process (ANP), Super Decisions, Training ship, Criteria and Sub-criteria

\section{INTRODUCTION}

$\mathrm{KRI}$ Dewaruci is a training ship for the Naval Academy Cadets to form strong prospective Navy officers. Having the spirit of the Pancasila Warriors and Sapta Marga, possessing the knowledge and professional skills of the military in the spectrum of initial assignments at KRI / Troops, and having leadership as a prospective Indonesian National Armed Forces (TNI) / Navy leader in the future. KRI Dewaruci measuring 58.5 meters and 9.5 meters wide from this Barquentine class was built in H.C. Stulchen \& Sohn Hamburg, West Germany. The KRI was launched on January 24, 1953. The age of the old KRI Dewaruci was very vulnerable to various threats of accidents while carrying out the voyage. The government with this consideration is planning to replace KRI Dewaruci as AAL cadets with a new training ship.

The selection of truly appropriate sailing boat alternatives requires analysis of information and identification of important requirements regarding data from alternative training ship. Which broadly covers operational requirements and technical requirements of training ship in the selection of procurement of replacement KRI Dewaruci.

By paying attention to the conditions and reality in the field, a major problem can be formulated in the discussion of this paper are how to determine the alternative training ship to procure a new training ship replacement for KRI Dewaruci and how to determine the main/critical criteria for training ship selection.

The purposes of this study are Determine the priority of new training ship alternatives for $A A L$ Cadets that are truly appropriate according to operational requirements and technical requirements by using the Analytical Network Process (ANP) method and Knowing the main sub criteria on the criteria of operational requirements and technical requirements.

\section{MATERIAL AND METHOD}

\subsection{Multi-Criteria Decision Making (MCDM)}

Mangkusubroto and Trisnadi (1983) stated that in this life, humans are always faced with various problems and issues. One problem that is 
certain and experienced by humans is how to make an appropriate decision on various choices (alternatives) and criteria (attributes) that exist.[1] Ciptomulyono (2010) gives an understanding of MCDM is an alternative process selection method to obtain optimal solutions from several alternatives. Problems with many criteria may be defined as a situation where a criterion is a consideration for choosing an alternative that is used to:

- Determine the best alternative or a set of the best alternatives (choice problems).

- Ranking alternatives from the best to the worst (ranking issues).

- $\quad$ Divide alternative sets into alternative subset based on several rules (sorting problems).[2]

\subsection{Analytic Network Process (ANP)}

Saaty (2001) explains the Analytic Network Process (ANP) is a method that produces a framework to overcome decision-making problems without involving assumptions related. It's independence between higher level elements with weak and independent elements at one level.[3] The pair comparison process uses a scale that reflects the importance of a decision element with other decision elements at the same hierarchy level. In the following Table 2.1, the following shows the scale of pair comparisons.

Table 1. Pair Comparison Scale

\begin{tabular}{|c|l|}
\hline Interest level & \multicolumn{1}{c|}{ Definition } \\
\hline 1 & Both elements are equally important \\
\hline 3 & One element is slightly more important than the other elements \\
\hline 5 & One element is actually more important than the other elements \\
\hline 7 & One element is clearly more important than the other elements \\
\hline 9 & One absolute element is more important than other elements \\
\hline $2,4,6,8$ & Middle values between 2 adjoining assessments \\
\hline
\end{tabular}

The advantage of ANP compared to AHP is that ANP frees the need to compile components in the form of straight chains as in the hierarchy. And by entering dependencies, feedback, and cycles of influence on the supermatrix, ANP is more objective and more likely to capture what is happening in the real world. Overall ANP is a better decision-making tool than $\mathrm{AHP}$, but $\mathrm{ANP}$ requires more work to capture facts and interactions.

This feedback structure does not have a straight top to bottom shape like in a hierarchy but rather resembles a network with a cycle that connects the components inside the component itself. An example of a feedback network structure can be seen in Figure 1.

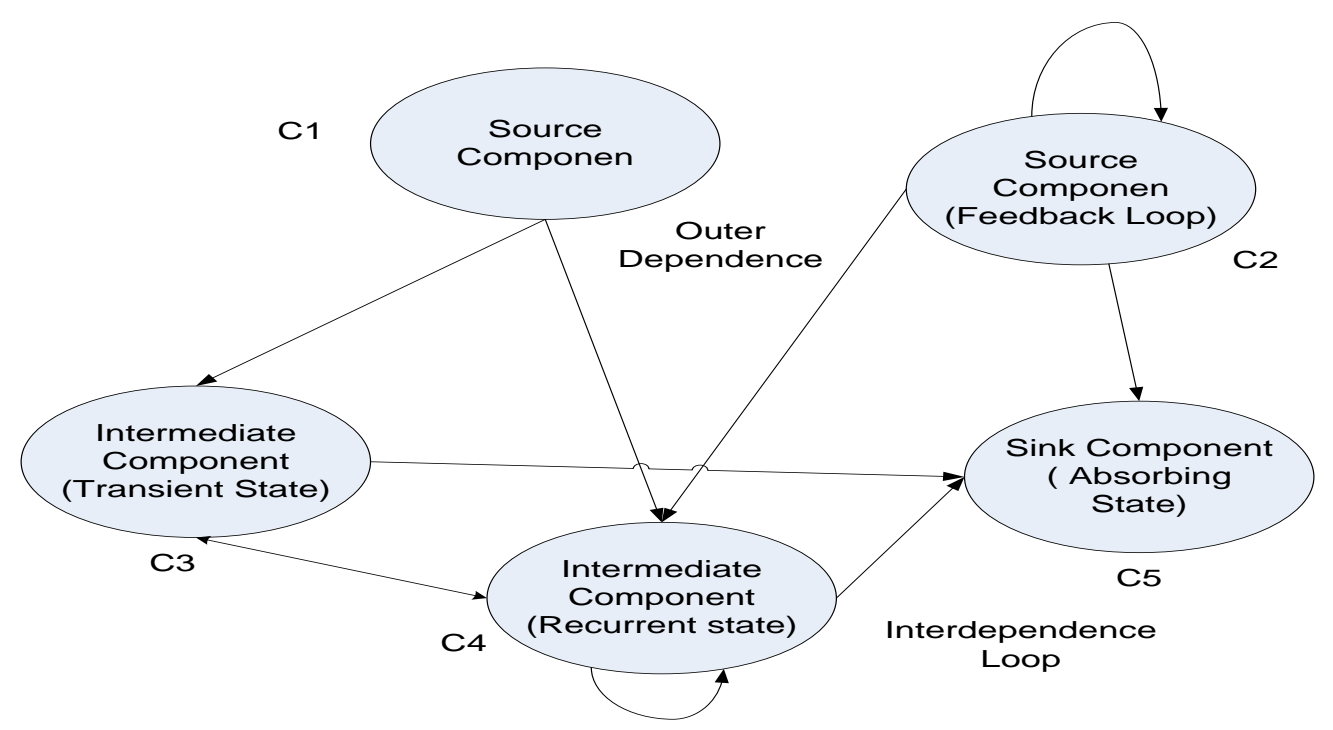

Fig.1 Feedback Network Structure 


\subsection{Operational Requirement}

Based on the Chief Operations Letter Number: R / 458 / VII / 2012 dated July 11, 2012, concerning the determination of the Operational Requirement of training ship intended as a guideline for the preparation of technical specifications to procure training sailing ship to complete further policy decisions. Some considerations in determining operational requirements include security, training, skills, transfer of technology, geographic conditions.[4]

\subsection{Technical Requirement}

Based on the Chief Logistics Staff Letter Number: R / 555 / VII / 2012 dated July 3, 2012, concerning the determination of the Technical Requirements to be explained as technical specifications that have been reviewed. It by the TNI Headquarters Planning and Logistics Staff, among others: Navigation, Training Equipment, Platforms, Machinery, Mast and Sail.[4]

\subsection{Research Flow Chart}

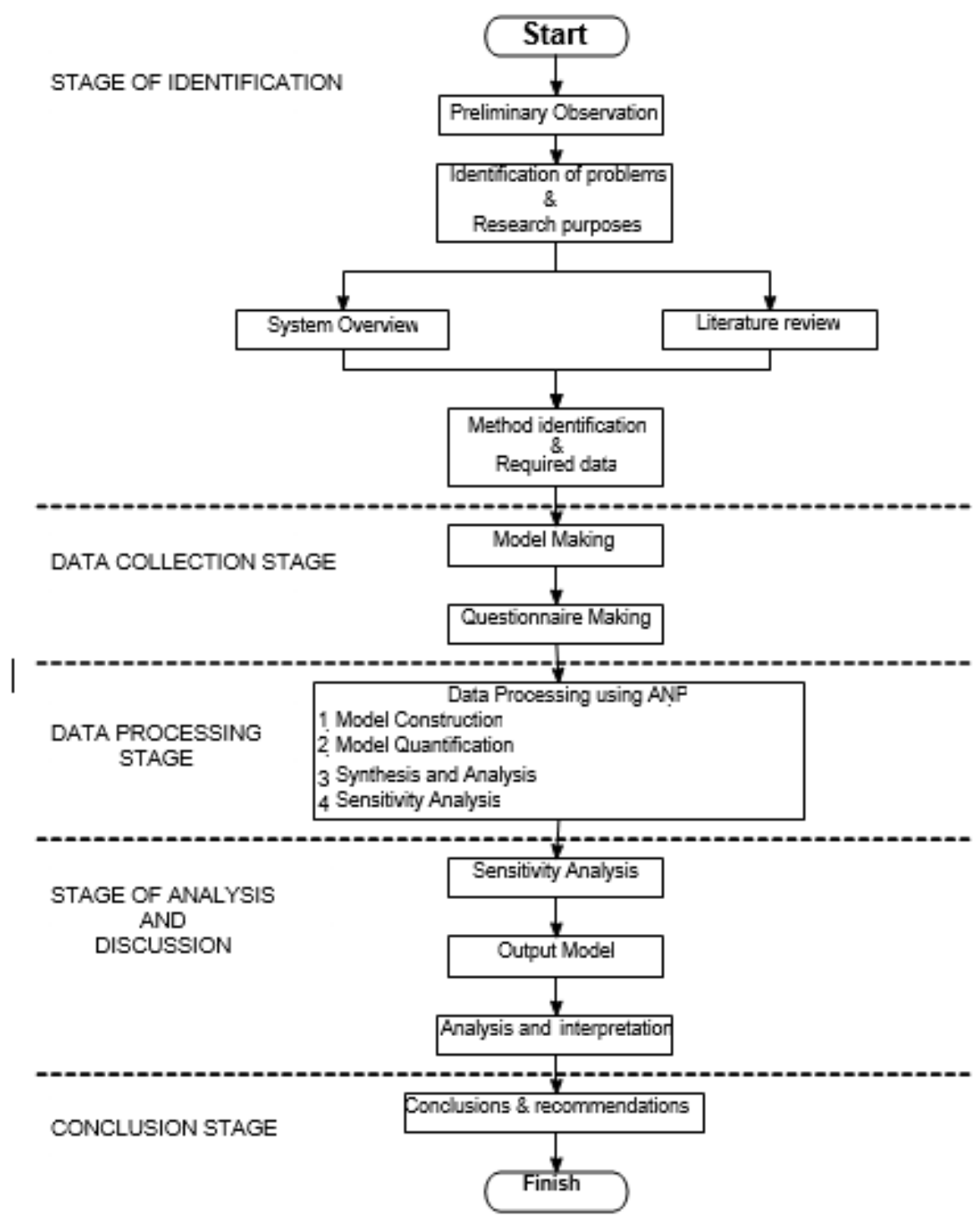

Fig.2 Research Flowchart 


\subsection{Determination of Criteria}

The criteria used for the selection of training ship were obtained based on the Warrant Number Sprin / 840 / VII / 2010 dated July 14, 2010, regarding the establishment of the Spectrum Evaluation Team for the procurement of replacement KRI Dewaruci and the Training ship

Procurement Study from Mabesal.[5]

Table 2. Criteria

\begin{tabular}{|c|c|l|}
\hline NO & \multicolumn{1}{|c|}{ CRITERIA } & \multicolumn{1}{|c|}{ DEFINITION/ ASSESSMENT PARAMETERS } \\
\hline 1 & Operational Requirements & $\begin{array}{l}\text { Operational requirements related to the strategic } \\
\text { value of training ship as navigation and nautical } \\
\text { training tool for AAL Cadets. }\end{array}$ \\
\hline 2 & Technical Requirements & $\begin{array}{l}\text { Technical requirements relating to the design and } \\
\text { technical specifications of training ships. }\end{array}$ \\
\hline
\end{tabular}

Table 3. Sub criteria on the Operational Requirements Criteria

\begin{tabular}{|c|l|l|}
\hline NO & SUB CRITERIA & \multicolumn{1}{|c|}{ DEFINITION / ASSESSMENT PARAMETERS } \\
\hline 1 & Security & $\begin{array}{l}\text { This training ship is used for AAL Cadet training facilities as a } \\
\text { future TNI leader so that it takes a very high safety and } \\
\text { security factor for the ship. It to minimize the accident factor } \\
\text { while carrying out the voyage. }\end{array}$ \\
\hline 2 & Skills Training & $\begin{array}{l}\text { As a means of AAL Cadet training in the field of navigation } \\
\text { and nautical as well as other additional functions such as } \\
\text { diplomacy functions, tourism ambassadors, limited patrols, } \\
\text { and limited maritime intelligence functions, a training ship is } \\
\text { needed to provide all supporting equipment. }\end{array}$ \\
\hline 3 & $\begin{array}{l}\text { Transfer of } \\
\text { Technology } \\
\text { (TOT) }\end{array}$ & $\begin{array}{l}\text { The construction of training ship must consider the Transfer of } \\
\text { Technology process so that in the future the Indonesian } \\
\text { nation will be able to build and maintain training ship } \\
\text { independently. }\end{array}$ \\
\hline 4 & $\begin{array}{l}\text { Geographical } \\
\text { Conditions }\end{array}$ & $\begin{array}{l}\text { Can operate across all Indonesian waters, oceans in the } \\
\text { world, and be able to stop at all ports in all countries in the } \\
\text { world and be able to sail on sea conditions more than sea } \\
\text { state 7 (Beaufort scale). }\end{array}$ \\
\hline
\end{tabular}

Table 4. Sub criteria on Criteria Technical Requirements

\begin{tabular}{|c|c|c|}
\hline NO & SUB CRITERIA & DEFINITION / ASSESSMENT PARAMETERS \\
\hline 1 & Navigation & $\begin{array}{l}\text { A navigation system that provides security assurance with } \\
\text { high accuracy navigation, both flat navigation, astronomical } \\
\text { navigation and electronic navigation. }\end{array}$ \\
\hline 2 & $\begin{array}{l}\text { Training } \\
\text { Equipment }\end{array}$ & $\begin{array}{l}\text { Has a complete marine equipment workshop that serves as a } \\
\text { training tool for AAL Cadets that can be used as a means of } \\
\text { repairing sails and other marine equipment on board. }\end{array}$ \\
\hline 3 & Platform & $\begin{array}{l}\text { Able to operate on sea conditions (Beaufort scale), have } \\
\text { nautical lifeboats, special rooms/classrooms, training } \\
\text { platforms that are separate from the main platform. AAL Cadet } \\
\text { accommodation facilities that are separate from ship crew } \\
\text { (sleeping room, dining room, recreation room, room shower), } \\
\text { a lounge accommodation and multipurpose deck. }\end{array}$ \\
\hline 4 & Machinery & $\begin{array}{l}\text { Having high maneuverability both when using engine } \\
\text { propulsion, sails or a combination of both, Able to sail } \\
\text { continuously at sea for } 30 \text { days with a combination of engine } \\
\text { and sail boosters. }\end{array}$ \\
\hline 5 & Masts and Sails & $\begin{array}{l}\text { Has } 3 \text { high masts by the design. Has sailed with international } \\
\text { competition standards that are capable of moving the ship at a } \\
\text { speed of } 15 \text { knots in wind blowing conditions on the } 7 \text { scale } \\
\text { sea state of Beaufort. }\end{array}$ \\
\hline
\end{tabular}




\subsection{Alternative Determination}

Based on the Auction Announcement Letter Number B / 24 / VIII / 2012 / PAN dated August 18, 2012, there are 5 shipyard companies from 3 countries registering themselves to bid. But after the factual verification of the shipyard company by the Procurement Committee Team, currently, there are only three shipyard companies from two countries.

Table 5. Alternative Prospective Training Ship Providers

\begin{tabular}{|c|l|c|}
\hline NO & \multicolumn{1}{|c|}{ SHIPYARD } & COUNTRY \\
\hline 1 & Piere Shipyard/ PT. Sinar Kokoh Persada & Spain \\
\hline 2 & Bumar Shipyard/ PT. Puramas Militerrindo & Poland \\
\hline 3 & Astileros Gondan Shipyard/ PT. Citra Persada & Spain \\
\hline
\end{tabular}

\section{RESULT AND DISCUSSION}

\section{1. $\quad$ ANP Network Modeling}

After determining the existing criteria and prospective provider alternatives, it is then formed into an ANP network model as shown in Figure 3 below:

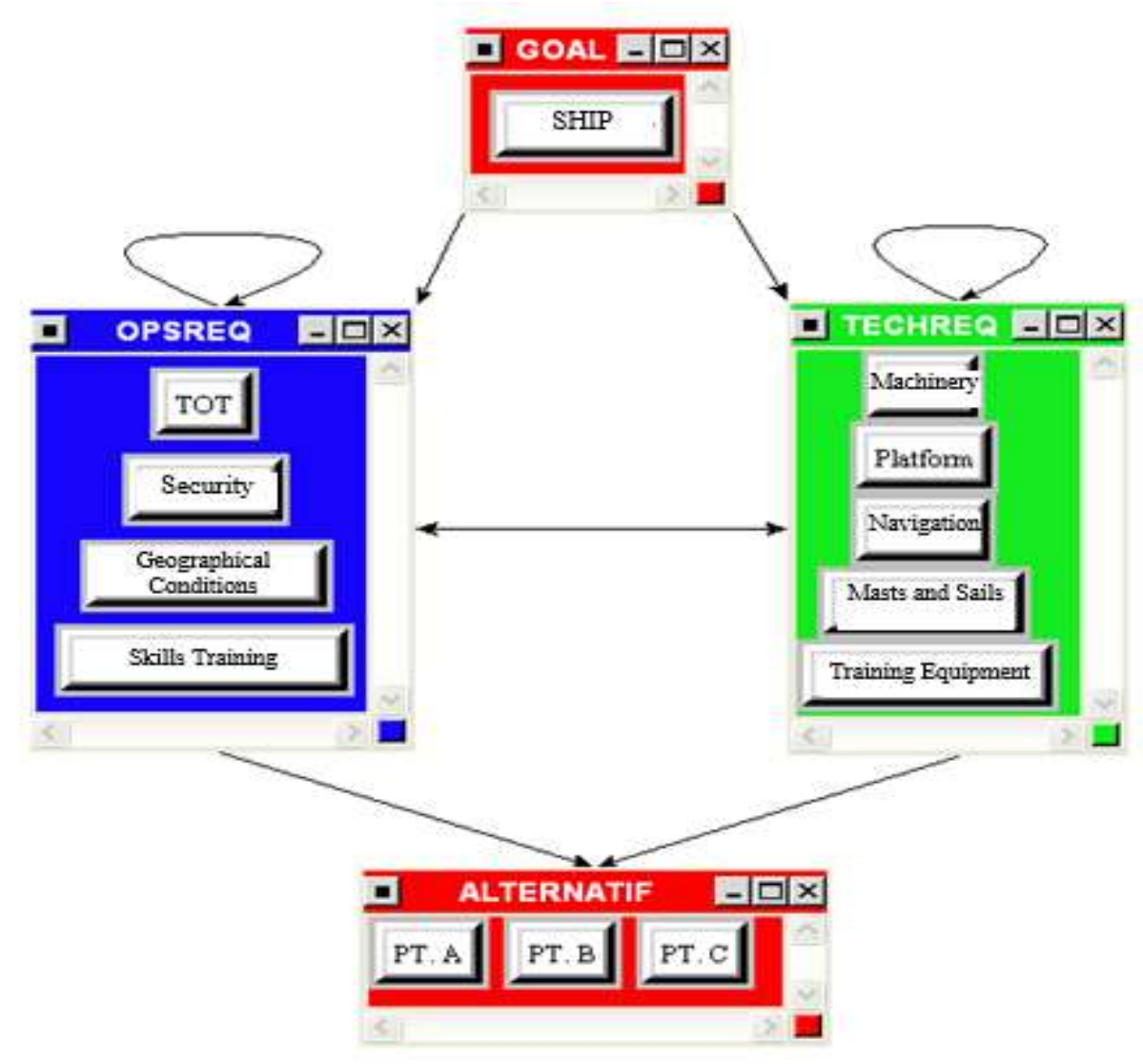

Fig.3 ANP Network Model Using Super Decisions Software Innerdependence

Table 6. Innerdependence Relations in the Cluster Operational Requirement

\begin{tabular}{|c|l|l|}
\hline NO & \multicolumn{1}{|c|}{ INNERDEPENDENCE } & \multicolumn{1}{c|}{ DEFINITION / ASSESSMENT PARAMETERS } \\
\hline 1 & TOT - Security & $\begin{array}{l}\text { With good TOT, domestic development can be achieved so it } \\
\text { can match the safety factor of products from abroad. }\end{array}$ \\
\hline
\end{tabular}




\begin{tabular}{|c|l|l|}
\hline NO & \multicolumn{1}{|c|}{ INNERDEPENDENCE } & \multicolumn{1}{|c|}{ DEFINITION / ASSESSMENT PARAMETERS } \\
\hline 2 & $\begin{array}{l}\text { TOT - Geographical } \\
\text { Conditions }\end{array}$ & $\begin{array}{l}\text { With the existence of TOT which is well sought at domestically, } \\
\text { it can develop products that can be adapted to international } \\
\text { geographical conditions. }\end{array}$ \\
\hline 3 & TOT - Skills Training & $\begin{array}{l}\text { TOT personnel is sought to provide their own skills training in } \\
\text { the future. }\end{array}$ \\
\hline 5 & $\begin{array}{l}\text { Security - Geographical } \\
\text { Conditions }\end{array}$ & $\begin{array}{l}\text { Geographical conditions vary in international waters so that } \\
\text { maximum safety factors are needed. }\end{array}$ \\
\hline 6 & Security - Skills Training & $\begin{array}{l}\text { Good skills training for Indonesian Navy personnel will increase } \\
\text { professionalism in safeguarding security factors. }\end{array}$ \\
\hline
\end{tabular}

Table 7. Innerdependence Relationship on Cluster Technical Requirements

\begin{tabular}{|c|c|c|}
\hline NO & INNERDEPENDENCE & DEFINITION/ ASSESSMENT PARAMETERS \\
\hline 1 & Machinery - Platform & $\begin{array}{l}\text { Machinery requires the design and specification of space in its } \\
\text { placement. }\end{array}$ \\
\hline 2 & Machinery - Navigation & $\begin{array}{l}\text { Existing navigation equipment influences the type and ability of } \\
\text { an auxiliary machine that produces electricity on the ship. }\end{array}$ \\
\hline 3 & $\begin{array}{l}\text { Machinery - Masts \& } \\
\text { Sails }\end{array}$ & Machinery and Sails can work together or individually at work. \\
\hline 4 & $\begin{array}{l}\text { Machinery - Training } \\
\text { Equipment }\end{array}$ & $\begin{array}{l}\text { Available training equipment must be supported by reliable } \\
\text { Machinery. }\end{array}$ \\
\hline 5 & Platform - Navigation & $\begin{array}{l}\text { Navigation equipment requires the design and specification of } \\
\text { space in its placement. }\end{array}$ \\
\hline 6 & Platform - Masts \& Sails & $\begin{array}{l}\text { Masts and Sails require the design and specification of space in } \\
\text { its placement. }\end{array}$ \\
\hline 7 & $\begin{array}{l}\text { Platform - Training } \\
\text { Equipment }\end{array}$ & $\begin{array}{l}\text { Existing training equipment requires the design and specification } \\
\text { of space in its placement. }\end{array}$ \\
\hline 8 & Navigation - Mast \& Sails & Navigation equipment will support the operation of the sails. \\
\hline 9 & $\begin{array}{l}\text { Navigation Training } \\
\text { Equipment }\end{array}$ & $\begin{array}{l}\text { Good training equipment also supports the provision of accurate } \\
\text { data in navigation. }\end{array}$ \\
\hline 10 & $\begin{array}{l}\text { Mast \& Sails - Training } \\
\text { Equipment }\end{array}$ & $\begin{array}{l}\text { Existing training equipment can assist in the operation of the } \\
\text { sails. }\end{array}$ \\
\hline
\end{tabular}

\subsubsection{Outerdependence}

In addition to the Innerdependence relationship that occurs in each cluster. There is also an Outerdependence relationship between the sub-criteria among the clusters.

Table 8. Outerdependence relationship between Inter-Cluster Criteria

\begin{tabular}{|c|c|c|c|}
\hline NO & $\begin{array}{c}\text { SUB } \\
\text { CRITERIA }\end{array}$ & $\begin{array}{c}\text { OUTER } \\
\text { DEPENDENCE }\end{array}$ & DEFINITION/ASSESSMENT PARAMETERS \\
\hline \multirow{5}{*}{1} & \multirow{5}{*}{ TOT } & - Security & TOT affects the safety of products made domestically. \\
\hline & & $\begin{array}{l}\text { - Geographical } \\
\text { Conditions }\end{array}$ & $\begin{array}{l}\text { In the TOT process must reckon the geographical } \\
\text { conditions of Indonesian waters. }\end{array}$ \\
\hline & & - Skills Training & $\begin{array}{l}\text { TOT must carry out the entire process of skills training for } \\
\text { each ship crew. }\end{array}$ \\
\hline & & - Platform & Maximum use of own country expertise in the platform \\
\hline & & $\begin{array}{l}\text { - Training } \\
\text { Equipment }\end{array}$ & Maximum utilization of the country's experts in the platform. \\
\hline
\end{tabular}




\begin{tabular}{|c|c|c|c|}
\hline NO & $\begin{array}{l}\text { SUB } \\
\text { CRITERIA }\end{array}$ & $\begin{array}{c}\text { OUTER } \\
\text { DEPENDENCE }\end{array}$ & DEFINITION/ASSESSMENT PARAMETERS \\
\hline \multirow{4}{*}{2} & \multirow{4}{*}{ Security } & $\begin{array}{l}\text { - Geographical } \\
\text { Conditions }\end{array}$ & $\begin{array}{l}\text { Shipping security must cover all geographical conditions of } \\
\text { the world's waters. }\end{array}$ \\
\hline & & - Skills Training & $\begin{array}{l}\text { In the training, the process must pay attention to security } \\
\text { factors. }\end{array}$ \\
\hline & & - Platform & The use of good ship materials to support safety factors. \\
\hline & & $\begin{array}{l}\text { - Training } \\
\text { Equipment }\end{array}$ & Training equipment must be safe for use by ship personnel. \\
\hline \multirow{2}{*}{3} & \multirow{2}{*}{$\begin{array}{l}\text { Geographical } \\
\text { Conditions }\end{array}$} & - Platform & $\begin{array}{l}\text { The platform must be strong by the conditions of the } \\
\text { geographical waters of the world. }\end{array}$ \\
\hline & & - Navigation & $\begin{array}{l}\text { Navigation equipment must be usable under any } \\
\text { geographical conditions. }\end{array}$ \\
\hline \multirow{4}{*}{4} & \multirow{4}{*}{ Skills Training } & - Machinery & $\begin{array}{l}\text { Skills training must be thoroughly Machinery and ship's } \\
\text { engines }\end{array}$ \\
\hline & & - Navigasi & Personnel must master navigation in the training process. \\
\hline & & - Masts \& Sails & $\begin{array}{l}\text { The skill of opening and closing the sails perfectly during } \\
\text { training. }\end{array}$ \\
\hline & & $\begin{array}{l}\text { - Training } \\
\text { Equipment }\end{array}$ & Training skills for all sailing equipment. \\
\hline \multirow{4}{*}{5} & \multirow{4}{*}{ Machinery } & $\begin{array}{l}\text { - Geographical } \\
\text { Conditions }\end{array}$ & $\begin{array}{l}\text { Procurement of good Machinery to support the shipping } \\
\text { process of changing geographical conditions. }\end{array}$ \\
\hline & & - Skills Training & $\begin{array}{l}\text { The Machinery is easily operated by ship personnel during } \\
\text { skills training. }\end{array}$ \\
\hline & & - Platform & $\begin{array}{l}\text { The dimensions of the engine must be by the situation of } \\
\text { the ship body. }\end{array}$ \\
\hline & & - Navigation & $\begin{array}{l}\text { Navigation equipment must be supported by electricity by } \\
\text { the engine and diesel generator. }\end{array}$ \\
\hline \multirow{7}{*}{6} & \multirow{7}{*}{ Platform } & - Machinery & $\begin{array}{l}\text { Placement of the Machinery must match the space } \\
\text { available on the ship. }\end{array}$ \\
\hline & & - Navigation & $\begin{array}{l}\text { The placement of navigation equipment must be by the } \\
\text { ship's body. }\end{array}$ \\
\hline & & - Masts \& Sails & Masts and sails must balance with the ship. \\
\hline & & $\begin{array}{l}\text { - Training } \\
\text { Equipment }\end{array}$ & $\begin{array}{l}\text { Supporting equipment must be adapted to the available } \\
\text { training ship body spaces. }\end{array}$ \\
\hline & & $\begin{array}{l}\text { - Geographical } \\
\text { Conditions }\end{array}$ & $\begin{array}{l}\text { With varying and different geographical conditions, a strong } \\
\text { training ship body must be supported. }\end{array}$ \\
\hline & & - Security & $\begin{array}{l}\text { The security of shipping ships must be supported by a } \\
\text { strong ship body. }\end{array}$ \\
\hline & & - Masts \& Sails & $\begin{array}{l}\text { The use of the sails must be supported by qualified } \\
\text { navigation. }\end{array}$ \\
\hline 7 & Navigation & $\begin{array}{l}\text { - Training } \\
\text { Equipment }\end{array}$ & $\begin{array}{l}\text { Supporting training equipment does not interfere with ship } \\
\text { navigation equipment. }\end{array}$ \\
\hline
\end{tabular}

\subsubsection{Data Collection with Questionnaires}

Questionnaire making uses a reference network model that has been formed. In answering questions in this questionnaire, respondents do not need to do discrete scoring with numbers but only do intuitive through linguistic variables. However, about filling in this questionnaire, the researchers attached comparative data from the prospective suppliers of goods/services.

\subsubsection{Data processing}

The next stage after obtaining data is data processing activities. About the method used in this study, the ANP method was used and the data processing was carried out through the help of Super Decisions software. The processed data is questionnaire data which is the respondents' perceptions regarding the selection of training ship. 
Table 9. Geometric Average Recapitations of Pairwise Comparison Value

\begin{tabular}{|l|c|c|c|c|}
\hline \multicolumn{2}{|l|}{ Criteria } & Geomean & Matrix \\
\hline Operational requirement & vs & Technical requirement & 1,44 & 1,44 \\
\hline
\end{tabular}

\begin{tabular}{|c|c|c|c|c|}
\hline \multicolumn{2}{|c|}{ Operational requirement sub-criteria } & Geomean & Matrik \\
\hline Security & vs & Skills Training & 4,14 & 4,14 \\
\hline Security & vs & Transfer of Technology & 2,79 & 2,79 \\
\hline Security & vs & $\begin{array}{c}\text { Geographical } \\
\text { Conditions }\end{array}$ & 2,00 & 2,00 \\
\hline Skills Training & vs & Transfer of Technology & 0,47 & 2,15 \\
\hline Skills Training & vs & $\begin{array}{c}\text { Geographical } \\
\text { Conditions }\end{array}$ & 1,59 & 1,59 \\
\hline Transfer of Technology & vs & $\begin{array}{c}\text { Geographical } \\
\text { Conditions }\end{array}$ & 2,74 & 2,74 \\
\hline
\end{tabular}

\begin{tabular}{|c|c|c|c|c|}
\hline \multicolumn{2}{|l|}{ Technical requirement sub-criteria } & Geomean & Matrix \\
\hline Navigation & vs & Training Equipment & 3,91 & 3,91 \\
\hline Navigation & vs & Platform & 2,29 & 2,29 \\
\hline Navigation & vs & Machinery & 4,10 & 4,10 \\
\hline Navigation & vs & Masts and Sails & 3,00 & 3,00 \\
\hline Training Equipment & vs & Platform & 0,35 & 2,87 \\
\hline Training Equipment & vs & Machinery & 0,50 & 2,00 \\
\hline Training Equipment & vs & Mast and & 0,35 & 2,87 \\
\hline Platform & vs & Machils & 5,00 & 5,00 \\
\hline Platform & vs & Masts and Sails & 3,97 & 3,97 \\
\hline Machinery & vs & Masts and Sails & 3,30 & 3,30 \\
\hline
\end{tabular}

\begin{tabular}{|c|c|c|c|c|}
\hline Sub-criteria TOT & Geomean & Matrix \\
\hline Security & vs & $\begin{array}{c}\text { Geographical } \\
\text { Conditions }\end{array}$ & 3,89 & 3,89 \\
\hline Security & vs & Skills Training & 2,29 & 2,29 \\
\hline Geographical Conditions & vs & Skills Training & 0,47 & 2,12 \\
\hline Sub-criteria TOT & vs & Training Equipment & Geomean & Matrix \\
\hline Platform & \multicolumn{2}{|l}{} & 3,96 \\
\hline
\end{tabular}

\begin{tabular}{|l|c|c|c|c|}
\hline \multicolumn{2}{|l|}{ Sub-criteria Security } & Geomean & Matrix \\
\hline Geographical Conditions & vs & Skills Training & 3,68 & 3,68 \\
\hline
\end{tabular}

\begin{tabular}{|c|c|c|c|c|}
\hline \multicolumn{2}{|l|}{ Sub-criteria Security } & Geomean & Matrix \\
\hline Platform & vs & Training Equipment & 4,25 & 4,25 \\
\hline
\end{tabular}

\begin{tabular}{|c|c|c|c|c|}
\hline \multicolumn{2}{|l|}{ Sub-criteria Geographical Conditions } & Geomean & Matrix \\
\hline $\begin{array}{c}\text { Astileros Gondan } \\
\text { ( Spain) }\end{array}$ & vs & Navigation & 3,68 & 3,68 \\
\hline
\end{tabular}

\begin{tabular}{|c|c|c|c|c|}
\hline \multicolumn{2}{|l|}{ Sub-criteria Skills Training } & Geomean & Matrix \\
\hline Machinery & vs & Navigation & 0,22 & 4,50 \\
\hline Machinery & vs & Masts and Sails & 0,48 & 2,09 \\
\hline Machinery & vs & Training Equipment & 0,32 & 3,10 \\
\hline Navigasi & vs & Masts and Sails & 2,74 & 2,74 \\
\hline Navigasi & vs & Training Equipment & 1,71 & 1,71 \\
\hline Masts and Sails & vs & Training Equipment & 0,25 & 4,00 \\
\hline
\end{tabular}




\begin{tabular}{|c|c|c|c|c|}
\hline Sub-criteria Machinery & Geomean & Matrix \\
\hline Platform & vs & Navigation & 0,35 & 2,83 \\
\hline Sub-criteria Machinery & Gkills Training & 3,85 & 3,85 \\
\hline Geographical Conditions & vs & Geomean & Matrix \\
\hline
\end{tabular}

\begin{tabular}{|c|c|c|c|c|}
\hline \multicolumn{2}{|l|}{ Subcriteria Platform } & Geomean & Matrix \\
\hline Machinery & vs & Navigation & 0,30 & 3,30 \\
\hline Machinery & vs & Masts and Sails & 0,48 & 2,09 \\
\hline Machinery & vs & Training Equipment & 0,50 & 2,00 \\
\hline Navigation & vs & Masts and Sails & 3,94 & 3,94 \\
\hline Navigation & vs & Training Equipment & 4,72 & 4,72 \\
\hline Masts and Sails & vs & Training Equipment & 0,27 & 3,75 \\
\hline
\end{tabular}

\begin{tabular}{|c|c|c|c|c|}
\hline \multicolumn{2}{|l|}{ Sub-criteria Platform } & Geomean & Matrix \\
\hline Security & vs & $\begin{array}{c}\text { Geographical } \\
\text { Conditions }\end{array}$ & 4,39 & 4,39 \\
\hline
\end{tabular}

\begin{tabular}{|c|c|c|c|c|}
\hline \multicolumn{2}{|l|}{ Sub-criteria Navigation } & Geomean & Matrix \\
\hline Masts and Sails & vs & Training Equipment & 0,24 & 4,25 \\
\hline
\end{tabular}

\section{INTER ALTERNATIVE COMPARISON}

\begin{tabular}{|c|c|c|c|c|}
\hline \multicolumn{2}{|l|}{ Sub-criteria Security } & Geomean & Matrix \\
\hline $\begin{array}{c}\text { Astileros Gondan ( } \\
\text { Spain) }\end{array}$ & vs & $\begin{array}{c}\text { Bumar Shipyard } \\
\text { (Poland) }\end{array}$ & 1,00 & 1,00 \\
\cline { 2 - 5 } & vs & Piere Shipyard (Spain) & 1,17 & 1,17 \\
\hline Bumar Shipyard (Poland) & vs & Piere Shipyard (Spain) & 0,56 & 1,79 \\
\hline
\end{tabular}

\begin{tabular}{|l|c|c|c|c|}
\hline \multicolumn{2}{|l|}{ Sub-criteria Skills Training } & Geomean & Matrix \\
\hline \multirow{2}{*}{ Astileros Gondan (Spain) } & vs & $\begin{array}{c}\text { Bumar Shipyard } \\
\text { (Poland) }\end{array}$ & 0,50 & 2,01 \\
\cline { 2 - 5 } & vs & Piere Shipyard (Spain) & 0,21 & 4,76 \\
\hline Bumar Shipyard (Poland) & vs & Piere Shipyard (Spain) & 0,32 & 3,13 \\
\hline
\end{tabular}

\begin{tabular}{|c|c|c|c|c|}
\hline \multicolumn{3}{|l|}{ Sub-criteria TOT } & \multirow{2}{*}{$\begin{array}{c}\text { Geomean } \\
1,08\end{array}$} & \multirow{2}{*}{$\begin{array}{l}\text { Matrix } \\
1,08\end{array}$} \\
\hline Astileros Gondan (Spain) & vs & $\begin{array}{c}\text { Bumar Shipyard } \\
\text { (Poland) }\end{array}$ & & \\
\hline & vs & Piere Shipyard (Spain) & 1,17 & 1,17 \\
\hline Bumar Shipyard (Poland) & vs & Piere Shipyard (Spain) & 0,54 & 1,84 \\
\hline
\end{tabular}

\begin{tabular}{|c|c|c|c|c|}
\hline \multicolumn{2}{|l|}{ Sub-criteria Geographical Conditions } & Geomean & Matrix \\
\hline \multirow{2}{*}{$\begin{array}{c}\text { Astileros Gondan ( } \\
\text { Spain) }\end{array}$} & vs & $\begin{array}{c}\text { Bumar Shipyard } \\
\text { (Poland) }\end{array}$ & 1,26 & 1,26 \\
\cline { 2 - 5 } & vs & Piere Shipyard (Spain) & 1,71 & 1,71 \\
\hline Bumar Shipyard (Poland) & vs & Piere Shipyard (Spain) & 0,39 & 2,57 \\
\hline
\end{tabular}

\begin{tabular}{|c|c|c|c|c|}
\hline \multicolumn{3}{|l|}{ Sub-criteria Machinery } & Geomean & Matrix \\
\hline \multirow{2}{*}{ Astileros Gondan (Spain) } & vs & $\begin{array}{l}\text { Bumar Shipyard } \\
\text { (Poland) }\end{array}$ & 1,49 & 1,49 \\
\hline & vs & Piere Shipyard (Spain) & 1,26 & 1,26 \\
\hline Bumar Shipyard (Poland) & vs & Piere Shipyard (Spain) & 1,47 & 1,47 \\
\hline
\end{tabular}




\begin{tabular}{|c|c|c|c|c|}
\hline \multicolumn{3}{|l|}{ Sub-criteria Navigation } & Geomean & Matrix \\
\hline \multirow[t]{2}{*}{ Astileros Gondan (Spain) } & vs & $\begin{array}{l}\text { Bumar Shipyard } \\
\text { (Poland) }\end{array}$ & 0,31 & 3,19 \\
\hline & vs & Piere Shipyard (Spain) & 0,35 & 2,86 \\
\hline Bumar Shipyard (Poland) & vs & Piere Shipyard (Spain) & 1,96 & 1,96 \\
\hline \multicolumn{3}{|c|}{ Sub-criteria Mast and sails } & Geomean & Matrix \\
\hline \multirow[t]{2}{*}{ Astileros Gondan (Spain) } & vs & $\begin{array}{l}\text { Bumar Shipyard } \\
\text { (Poland) }\end{array}$ & 2,32 & 2,32 \\
\hline & vs & Piere Shipyard (Spain) & 0,39 & 2,59 \\
\hline Bumar Shipyard (Poland) & vs & Piere Shipyard (Spain) & 0,35 & 2,83 \\
\hline
\end{tabular}

\begin{tabular}{|l|c|c|c|c|}
\hline \multicolumn{2}{|l|}{ Sub-criteria Platform } & Geomean & Matrix \\
\hline \multirow{2}{*}{ Astileros Gondan (Spain) } & vs & $\begin{array}{c}\text { Bumar Shipyard } \\
\text { (Poland) }\end{array}$ & 1,38 & 1,38 \\
\cline { 2 - 5 } & vs & Piere Shipyard (Spain) & 1,36 & 1,36 \\
\hline Bumar Shipyard (Poland) & vs & Piere Shipyard (Spain) & 1,59 & 1,59 \\
\hline
\end{tabular}

\begin{tabular}{|l|c|c|c|c|}
\hline \multicolumn{2}{|l|}{ Sub-criteria Training Equipment } & Geomean & Matrix \\
\hline \multirow{2}{*}{ Astileros Gondan (Spain) } & vs & $\begin{array}{c}\text { Bumar Shipyard } \\
\text { (Poland) }\end{array}$ & 1,61 & 1,61 \\
\cline { 2 - 5 } & vs & Piere Shipyard Spain & 1,26 & 1,26 \\
\hline Bumar Shipyard (Poland) & vs & Piere Shipyard (Spain) & 1,71 & 1,71 \\
\hline
\end{tabular}

Geometric averages that have been calculated are then entered into pairwise comparison matrices in software super decisions.

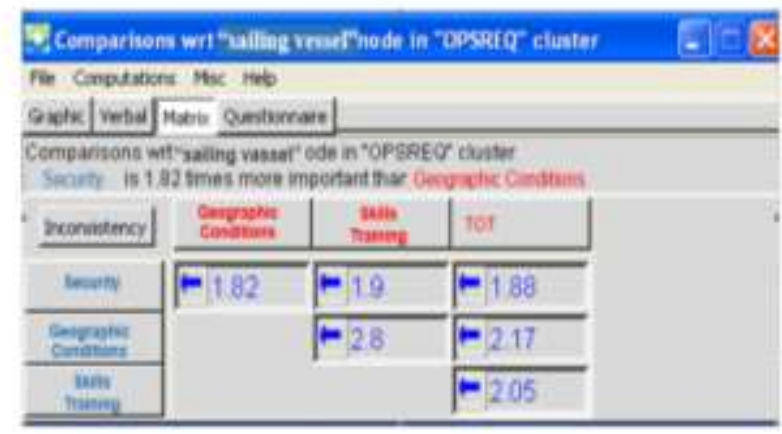

Fig.4 Pair Comparison Matrix

After obtaining a pairwise comparison value for each relationship a local priority score calculation is performed. Every time a local priority scoring should not exceed the value of 0.1 . For example, it can be seen in Figure 4.5 which shows the value of inconsistencies from paired comparisons between sub-criteria in the Operational Requirement criteria.

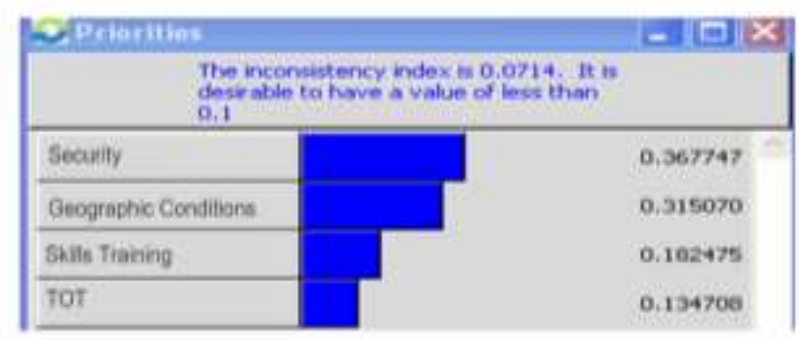

Fig.5 Inconsistency Index Among Sub Criteria

\subsection{Processing with Super Decisions Software}

After entering all geometric mean into the matrix format in the Super Decisions software, the software performs all stages of the ANP method by running Priorities. Which contains an alternative score and all sub-criteria score as shown in Fig 6. 


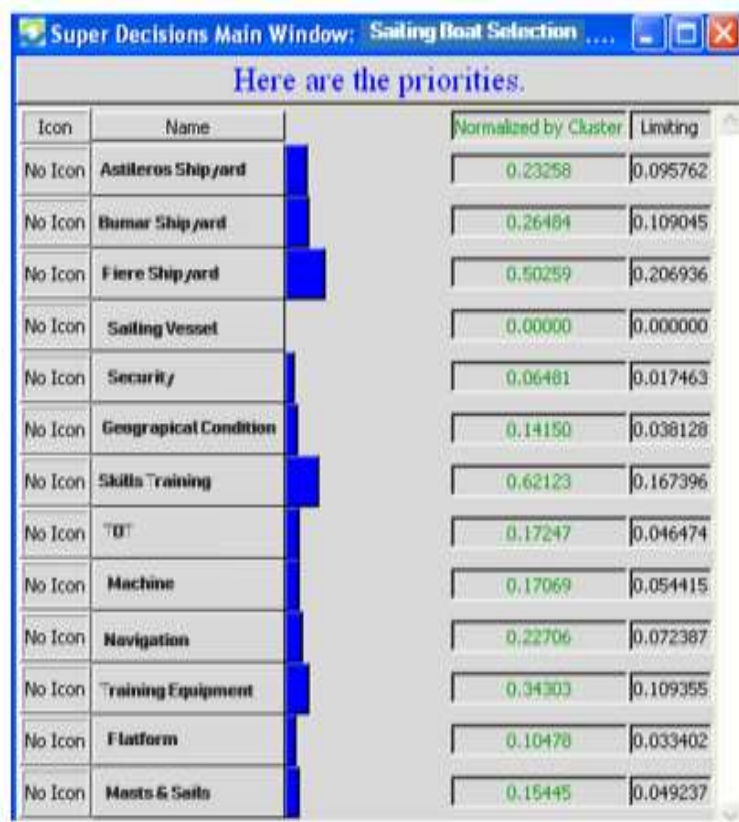

Fig.6 Final Score of Priorities for Selection of Training Ship

Furthermore, the final results in the form of rankings from sub-criteria in alternative groups can be seen by way of Synthesise in software super decisions as shown in Figure 7.

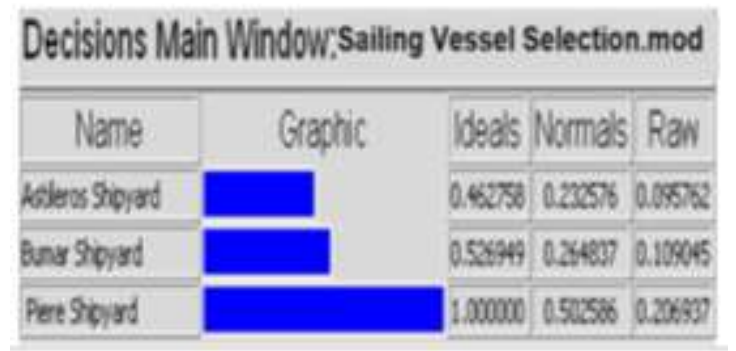

Fig.7 Final Results of Selection of Training Ship

\subsection{Sensitivity Analysis}

Sensitivity analysis was performed using the Super Decisions software by changing the scoring criteria for the tested alternatives. In this test, it can be seen that by changing the score of the criteria on the tested alternatives, it affects the results of the initial ranking or not. Whenever there is a point where there is a ranking/priority change, the point is called the critical point of an alternative.

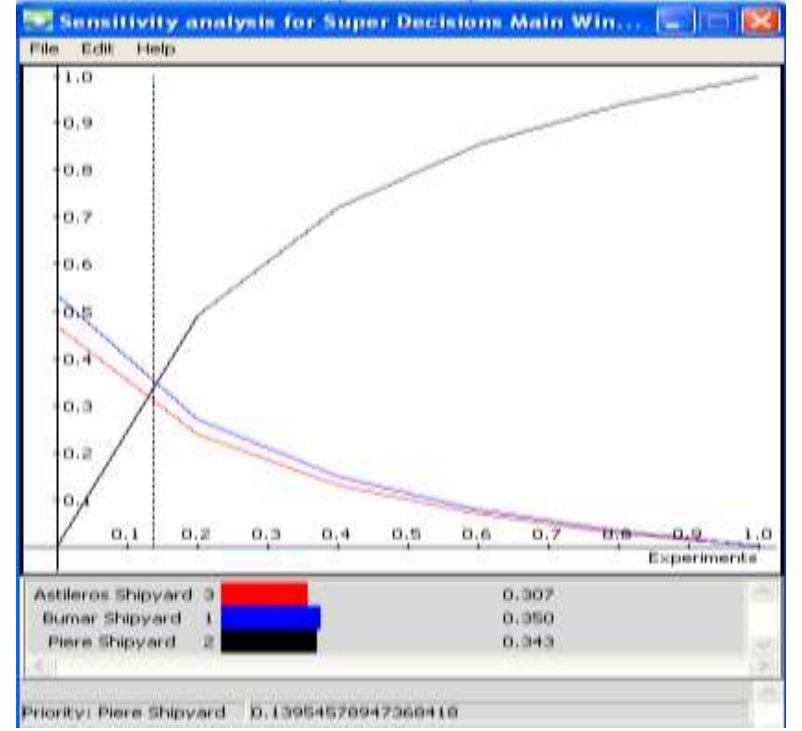

Fig.8 Sensitivity Analysis of Piere Shipyard

\subsection{Analysis of Training Ship Priority Alternative Ranking}

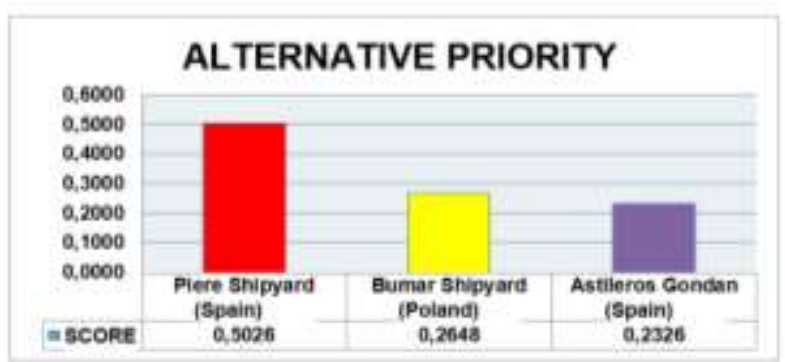

Fig.9 Alternative Priority Charts

Figure 9 shows that Piere Shipyard (Spain) is the shipyard with the highest score, followed by Bumar Shepard (Poland), and Astileros Shipyard (Spain). Thus Piere Shipyard (Spain) is a prospective provider of Substitute KRI Dewaruci getting the highest priority for Operational Requirements and Technical Requirements.

\subsection{Analysis of Operational Requirements and} Technical Requirements Criteria

On the alternative criteria for selecting a training ship, two criteria have been determined, namely the Operational Requirement and Technical Requirement. From the Super Decisions software in Figure 5.2 below, it can be seen that the Operational requirement criteria have a higher score, which is 0.630996 compared to the Technical 
requirement criteria with a score of 0.369004 . This illustrates that the Operational Requirement criteria are more influential than the Technical Requirement criteria.

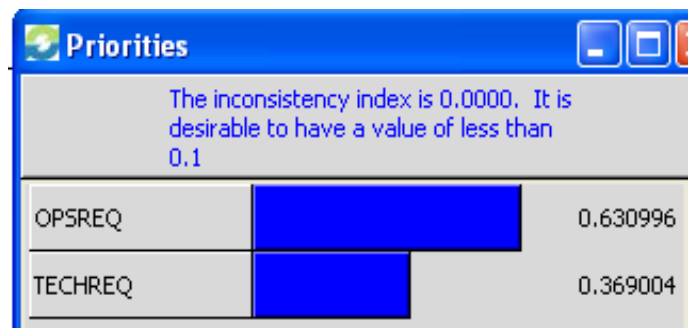

Fig.10 Value of Operational Requirements and Technical Requirements

The reason for the Operational Requirements criteria is more influential because in planning to procure a ship, the Operational Requirements criteria become a guideline and reference in making a Technical Requirement. If The Operational Requirements are made well and are made by competent experts in their field. The Technical Requirements provided will be by the Operational Requirements planning to produce a good ship.

\subsection{Sub-criteria Analysis in Operational Requirement Criteria}

The Operational requirements criteria are 4 sub-criteria, namely security, geographical conditions, skills training, and transfer of technology. The Skill Training sub-criteria have the highest score, which is 0.62123 compared to other sub-criteria. This illustrates that the Training Skills subcategory is the most influential compared to other sub-criteria.

\subsection{Sub-criteria Analysis in Technical Requirement Criteria}

In table 5.1, the criteria for the Technical Requirement are 5 sub-criteria, namely machine, navigation, training equipment, platform, masts, and sails. The Training Equipment sub-criteria have the highest score of 0.343030 compared to other subcriteria. This illustrates that the Training Tools subcategory is the most influential compared to other sub-criteria.

Table 10. Ranking of Alternative Subcriteria

\begin{tabular}{|c|l|c|c|c|}
\hline \multirow{3}{*}{ CRITERIA } & \multirow{2}{*}{ SUB CRITERIA } & \multicolumn{3}{|c|}{ ALTERNATIVE } \\
\cline { 3 - 5 } & $\begin{array}{c}\text { PIERE SHIPYARD } \\
\text { (SPAIN) }\end{array}$ & $\begin{array}{c}\text { BUMAR SHIPYARD } \\
\text { (POLAND) }\end{array}$ & $\begin{array}{c}\text { ASTILEROS SHIPYARD } \\
\text { (SPAIN) }\end{array}$ \\
\hline \multirow{3}{*}{$\begin{array}{c}\text { Operational } \\
\text { Requirement }\end{array}$} & Security & 0,419196 & 0,269859 & 0,310945 \\
\cline { 2 - 5 } & Geograpical condition & 0.509145 & 0.210102 & 0.280752 \\
\cline { 2 - 5 } & Skills Training & 0.648483 & 0.227376 & 0.124141 \\
\cline { 2 - 5 } & TOT & 0.421921 & 0.259915 & 0.318164 \\
\hline \multirow{4}{*}{$\begin{array}{c}\text { Technical } \\
\text { Requirement }\end{array}$} & Machinery & 0.403540 & 0.330098 & 0.266362 \\
\cline { 2 - 5 } & Navigation & 0.328285 & 0.533197 & 0.138518 \\
\cline { 2 - 5 } & Training Equipment & 0.417958 & 0.230889 & 0.351153 \\
\cline { 2 - 5 } & Platform & 0.421558 & 0.250862 & 0.327579 \\
\cline { 2 - 5 } & Masts \& Sails & 0.561813 & 0.157053 & 0.281134 \\
\hline
\end{tabular}

\subsection{Analysis of Alternative Sensitivity Tests}

The results of calculations that have been done illustrate an ideal situation. To anticipate changes the estimates that have been made before, a sensitivity analysis of these estimates is carried out. Sensitivity analysis is carried out to determine the extent of priority stability of the alternatives. The sensitivity test carried out can be said that the results of the selection of alternative training ship are not sensitive to changes in time and environment. 
Table 11. Sensitivity Test of Input Changes Value Score Skills Sub Criteria Training

\begin{tabular}{|c|c|c|c|c|}
\hline $\begin{array}{c}\text { MNPUT } \\
\text { VALUE }\end{array}$ & $\begin{array}{c}\text { SKILLS TRAINING } \\
\text { PIERE SHIPYARD } \\
\text { (SPAIN) }\end{array}$ & $\begin{array}{c}\text { ASTILEROS } \\
\text { SHIPYARD } \\
\text { (SPAIN) }\end{array}$ & $\begin{array}{c}\text { BUMAR } \\
\text { SHIPYARD } \\
\text { (POLAND) }\end{array}$ & $\begin{array}{c}\text { PIERE } \\
\text { SHIPYARD } \\
\text { (SPAIN) }\end{array}$ \\
\hline 0 & 1,00 & 31,05 & 40,76 & 28,20 \\
0,2 & 20,60 & 28,66 & 36,37 & 34,97 \\
0,4 & 40,20 & 26,27 & 31,99 & 41,74 \\
0,6 & 59,80 & 23,87 & 27,61 & 48,51 \\
0,8 & 79,40 & 21,48 & 23,23 & 55,29 \\
1 & 99,00 & 19,09 & 18,85 & 62,06 \\
\hline
\end{tabular}

Table 12. Alternative Sensitivity Test

\begin{tabular}{|l|c|c|c|c|}
\hline \multicolumn{1}{|c|}{ ALTERNATIVE } & $\begin{array}{c}\text { MINIMAL } \\
\text { VALUE }\end{array}$ & $\begin{array}{c}\text { VALUE OF } \\
\text { CHANGE }\end{array}$ & $\begin{array}{c}\text { MAXIMUM } \\
\text { VALUE }\end{array}$ & $\begin{array}{c}\text { CHANGE OF } \\
\text { PRIORITY }\end{array}$ \\
\hline Security & $0,00 \%$ & Infinity & $100,00 \%$ & Piere Shipyard \\
Geographical Condition & $0,00 \%$ & Infinity & $100,00 \%$ & Piere Shipyard \\
Skills Training & $0,00 \%$ & $22,90 \%$ & $100,00 \%$ & Bumar Shipyard \\
Transfer of Technology & $0,00 \%$ & Infinity & $100,00 \%$ & Piere Shipyard \\
Machinery & $0,00 \%$ & Infinity & $100,00 \%$ & Piere Shipyard \\
Navigation & $0,00 \%$ & Infinity & $100,00 \%$ & Piere Shipyard \\
Training Equipment & $0,00 \%$ & Infinity & $100,00 \%$ & Piere Shipyard \\
Platform & $0,00 \%$ & Infinity & $100,00 \%$ & Piere Shipyard \\
Masts and Sails & $0,00 \%$ & Infinity & $100,00 \%$ & Piere Shipyard \\
\hline
\end{tabular}

For the scoring of the Skill Training subcriteria, an alternative priority change occurs when the criteria score is lowered to $22.90 \%$. The changes that occur are the alternative Bumar Shipyard to be ranked first. While for the other subcriteria, the increase interval is infinity. Which means that the score can be increased to a maximum of score that is up to $100 \%$ and also scores can be reduced to a minimum score of $0 \%$ without changing the priority ranking.

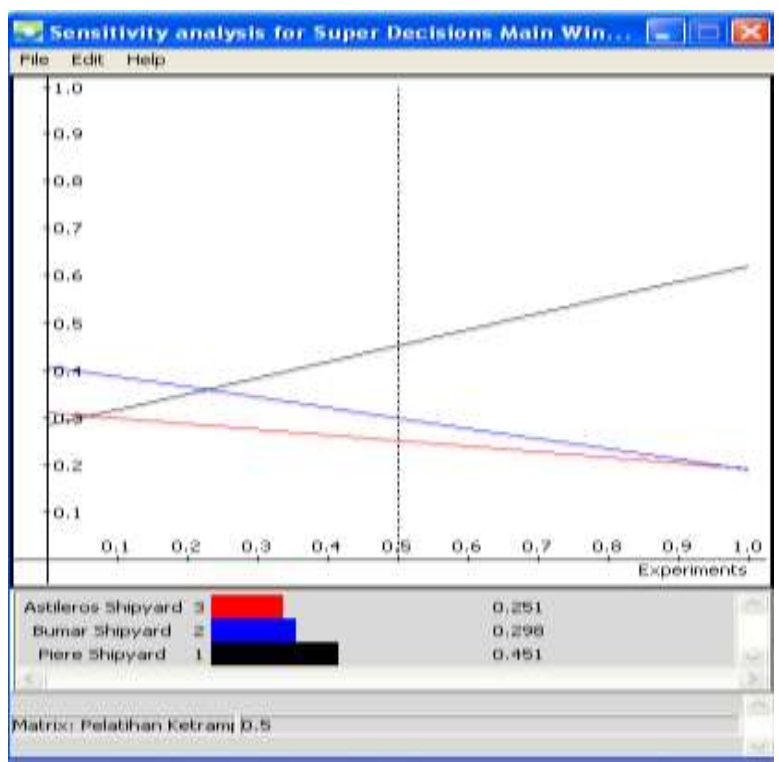

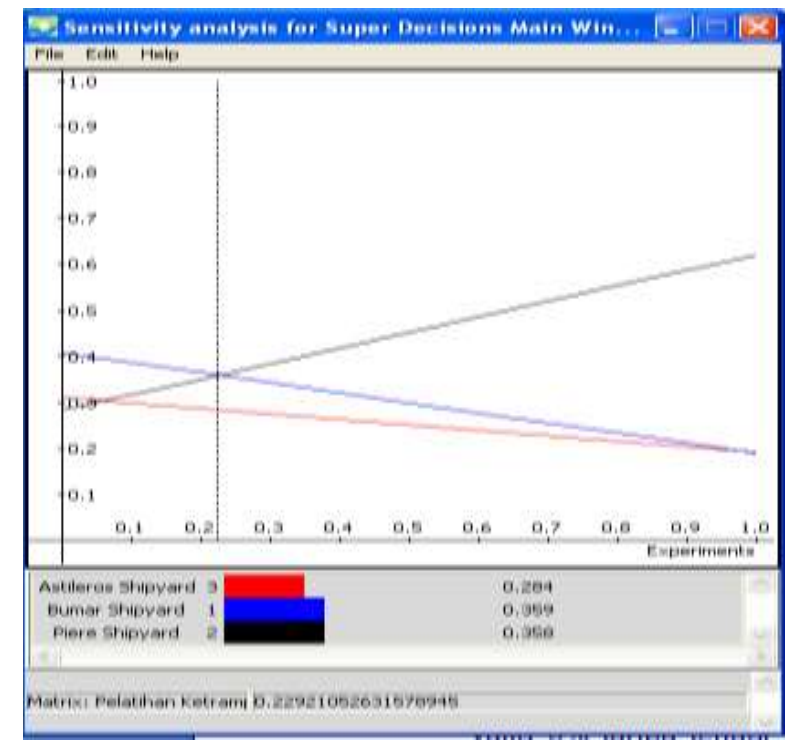

Fig.11 Alternative Priority Changes

The sensitivity test results can be explained that the left image is a picture before the score changes in the sub-criteria value of the Training Equipment are carried out. While the right picture is after the score changes are sub-criteria for training equipment. Sensitivity tests are carried out by increasing the score of 0.2 to 1 in the training equipment sub-criteria which is the main subcategory of the operational requirements criteria. 


\section{CONCLUSION}

The conclusions that can be taken in this study are:

a. From the results of data collection and processing, as well as the problem analysis of the selection of selected alternative training ship that get the highest priority score, namely Piere Shipyard (Spain) with a priority score of 0.502586 . Furthermore, in an alternative priority sequence in training ship selection is Bumar Shipyard (Poland) with a priority score of 0.264838 and Astileros Shipyard (Spain) with a priority score of 0.232676 .

b. In the Opsreq criterion, the Skills Training has the highest score, which is 0.62123 compared to other sub-criteria. This illustrates that the skills criteria sub-criteria are critical sub-criteria in operational requirements criteria.

c. The Techreq criteria for the Training Equipment sub-criteria have the highest score of 0.343030 compared to other sub-criteria. This illustrates that the subcategory of Training Equipment is a critical sub-criteria in the technical requirement criteria.

The following are suggestions for the Indonesian Navy and further research:

a. Suggestions for the Indonesian Navy, especially decision makers, are to pay more attention to the important and appropriate criteria in the procurement of a type of ship and to use the ANP method to solve complex preference problems.

b. For the next researchers to be able to combine software super decisions with a Decisions Support System based on management information systems in work units within the Indonesian Navy in solving complex problems.

\section{ACKNOWLEDGMENT}

This research has been supported by Indonesia Naval Technology College (STTAL), Indonesian Navy and Ministry of Defense of the Republic of Indonesia.

\section{REFERENCES}

Mangkusubroto dan Trisnadi. 1983. Decision Analysis: Systems Approach in Business and Project Management. Ganeca Exact. Bandung.

Ciptomulyono, U. 2010. The Multi-Criteria Decision Making Paradigm in the Perspective of Environmental and Project Development in Industry. Sepuluh Nopember Institute of Technology. Surabaya.

Saaty, T. L. 2001. Decision Making With Dependence and Feed Back: The Analytic Network Process. RWS Publication. Pittsburgh.

Mabesal. 2012. Determination of Operational and Technical Requirements for Procurement of Training Ship. Mabesal. Jakarta.

Mabesal. 2010. Establishment of Evaluation Team for Technical Specifications for the Procurement of Training Ship. Mabesal. Jakarta. 